\title{
Analysis of current harmonic on power system fuses using ANSYS
}

\author{
Hassan Feshki Farahani* and Masoud Sabaghi \\ Department of Electrical Engineering, Ashtian Branch, Islamic Azad University, Ashtian, Iran \\ hfeshki@yahoo.com*
}

\begin{abstract}
One of the most important protective devices in power systems are fuses which have been used for ages. Passing short circuit current from fuse causes to warm fuse element that it will open this path. Fuse temperature can directly affect fuse operation and its melting as well. Therefore, fuse should be designed in a way that it does not operate at nominal current and instantaneous over currents whereas it should operate at error case. In this paper, firstly, fuse element is investigated and its behavior is studied. Then, a sample fuse with its different sections is simulated under sinusoidal and non- sinusoidal currents. After that, different parameters such as thermal distribution, thermal flux, and electrical potential in all fuse parts are obtained for sinusoidal and non-sinusoidal currents. Also, Fuse temperature for various THD values is determined. Finally, an equation to determine fuse nominal current due to harmonics is presented.
\end{abstract}

Keywords: Thermal Flux, Current Harmonics, Power System Fuses, Finite Element Analysis, ANSYS.

\section{Introduction}

One of the earliest and simple protective devices in power systems are fuses which are classified into four categories (Torres, Fernandez et al., 2005 ): High-voltage (HV): (132 kV); Medium-voltage (MV): ( $1 \mathrm{kV}$ to $132 \mathrm{kV})$; Low-voltage (LV): (until $1 \mathrm{kV}$ ); Miniature: associated with physical dimensions.

In recent research, several models of fuses were developed (Dolegowski, 1976; Gnanalingam \& Wilkins 1980; Narancic \& Fecteau 1984; Petit, St-Jean et al., 1989; Bottauscio, 1991; Douglas, 1993; Lee, 2010), most of them are based on a mathematical representation of the arc physics. These models include transient heating and fusion of notched strip elements in sand, arc ignition, and subsequent burn-back, radial expansion of the arc channels due to fusion of the sand, merging of adjacent arcs, and many other second-order effects (Memiaghe, Bussière et al., 2007; Rochette, Bussière et al., 2007). Plesca (2007), developed a 3D thermal model in order to study the temperature distribution at a fast fuse. In this model, the thermal behavior of fast fuse depends on design of fuse link elements, material parameters and ambient conditions. Lindmayer (1999), has developed a Windows based program code for modeling complete fuses, including M-Effect, using the Finite Volume Method.

Beaujean, Newbery et al. (1995) has used a commercial FEM package to model heating of relatively simple fuse geometries without notches and with one single notch, respectively. Other FEM work has been reported in (Cañas, Fernández et al., 1999;

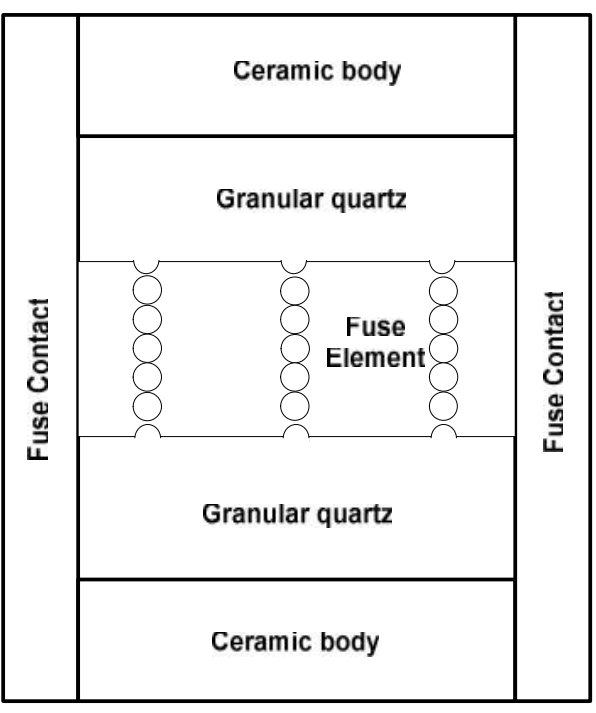

"Power system fuses" http://www.indjst.org
Jakubiuk \& W, 2003; Pleşca, 2003).

Wilkins (1991) described the temperature distribution and the thermal and electrical resistances of basic elements of the fuses by exact or semi-empirical analytical equations, and combined with iterative solution procedures. The fuse link is represented by an equivalent R-C network (Gelet, Tournier et al., 1999; Pleşca, 2001; Hoffmann \& Kaltenborn, 2003), Other simulations have also been done for fuse analysis among them finite element (Fernández, Cañas et al., 1995; Kürschner, Ehrhardt et al., 1995; Wilniewczyc, McEwan et al., 1999), or Finite Difference schemes (Garrido \& Cidrás, 1999) can be mentioned.

John (2006) presented thermal analysis of a medium voltage fuse by means of the finite element method. The thermal problem in electric fuses has been studied by different authors (Agarwal, Stokes et al., 1987; Sasu, 1995; Baraboi, Ciutea et al., 1999; Lijun, Zhiying et al., 1999; Kawase \& Miyatake, 2000; Hoffmann \& Kaltenborn, 2003), who have focused their analysis on the fuse element (Agarwal, Stokes et al., 1987; Sasu, 1995), the fuse contacts (Baraboi, Ciutea et al., 1999), or larger parts of the fuse (Lijun, Zhiying et al., 1999; Kawase \& Miyatake, 2000; Hoffmann \& Kaltenborn, 2003). In this paper, by modeling a 2D thermal model of fuse in ANSYS, the effect of harmonic on fuse thermal operation is investigated. The temperature distribution and thermal flux through all fuse link elements are obtained by ANSYS simulation.

Analysis of fuse operation for sinusoidal case

In this part, simulation is carried out for sinusoidal current. For this purpose, a fuse with following electrical 
Fig. 2. Temperature distribution along the fuse element

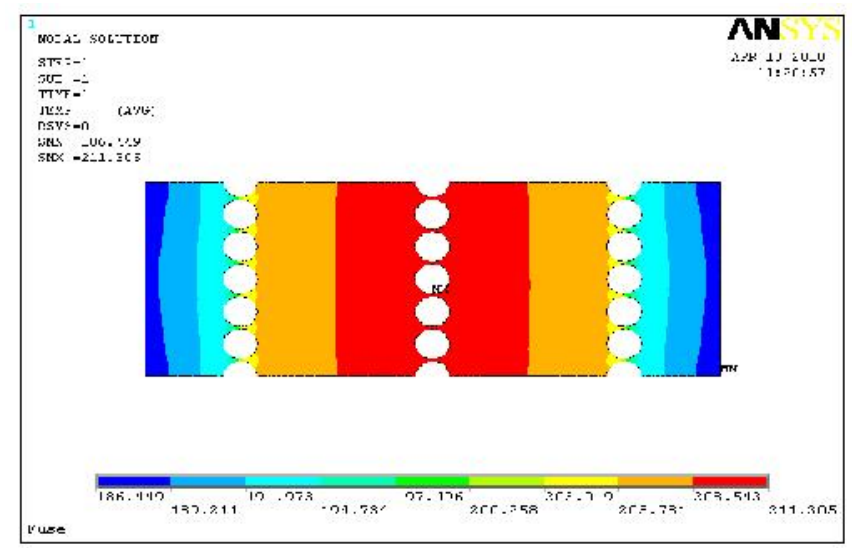

Fig. 3. Temperature distribution along the fuse length in sinusoidal condition

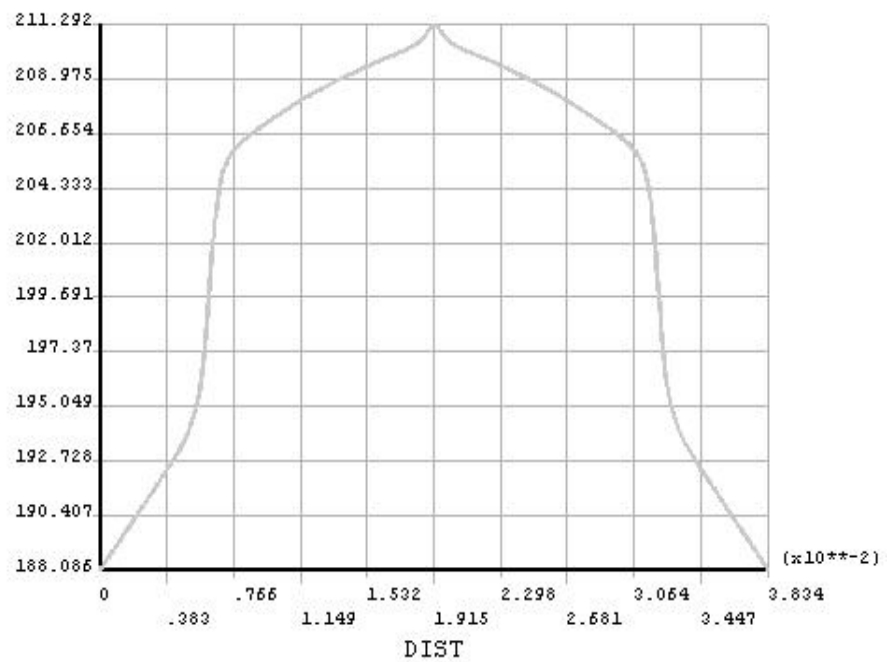

characteristics is simulated:

$$
I=100 \mathrm{~A}, \Delta V=22 \mathrm{mV}, R=0.22 \mathrm{~m} \Omega, T_{\text {melting }}=230{ }^{\circ} \mathrm{C}
$$

When fuse temperature reaches over $230{ }^{\circ} \mathrm{C}$, fuse will be melted. The sample fuse with approximate length of $40 \mathrm{~cm}$ is modeled in ANSYS which is shown in Fig. 1.

In this case, static analysis is used. Thermal distribution in fuse is shown in Fig. 2. According to this figure, the maximum temperature belongs to center of fuse element. The minimum temperature is in external edges of fuse element which its value is $186.449{ }^{\circ} \mathrm{C}$ and the center of fuse element has maximum temperature about $211.305{ }^{\circ} \mathrm{C}$. Temperature distribution versus fuse length is plotted in Fig. 3. In this figure, the maximum
Vol. 5 No. 3 (Mar 2012)

ISSN: 0974- 6846

Fig. 4. Voltage distribution along the fuse link

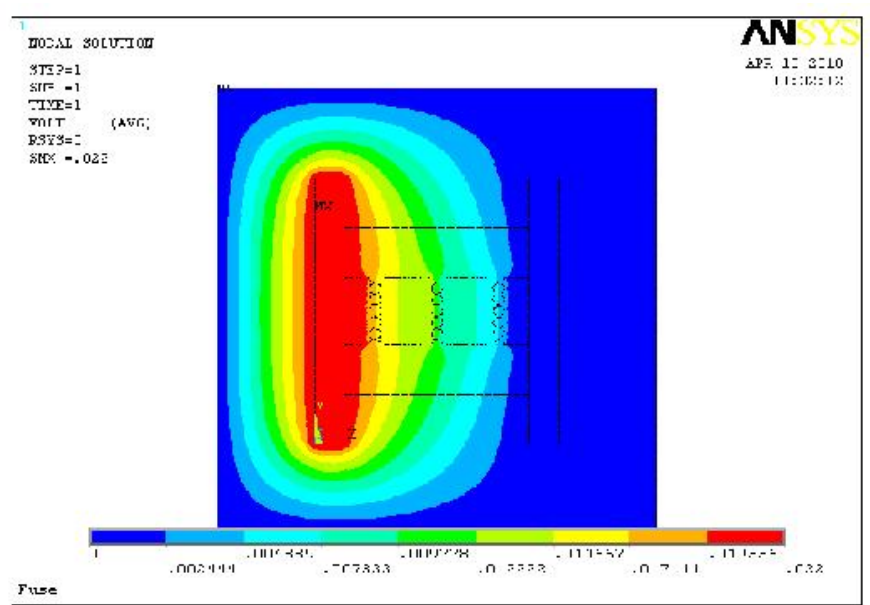

value is in fuse element centre as it is going far from center, temperature value is decreasing (Table 1).

Fig. 4 shows voltage distribution in fuse link. In this case, for each fuse contact, voltages of $0 \mathrm{~V}$ and $22 \mathrm{mV}$ are applied respectively. Voltage distribution through fuse element length is shown in Fig. 5. Considering this figure, the maximum and minimum fuse voltage values are respectively equal to $0.936 \mathrm{mV}$ and $21.069 \mathrm{mV}$. One of the other important parameters in fuse thermal analysis is thermal flux which for fuse element is plotted in Fig. 6. It can be viewed from this figure that the maximum thermal flux value is in flux element edges which is equal to $1.27 \times 10^{-7} \mathrm{~W} / \mathrm{m}^{2}$.

Fuse thermal behavior analysis using finite element for non- sinusoidal current

Here, it is assumed that fuse current waveform is nonsinusoidal. In other words, it contains harmonics. These harmonics affect fuse temperature rising which will be investigated in this part. First, the effects are discussed. Then, for different cases (current with different THD values), fuse will be simulated with ANSYS.

\section{Losses in fuse element}

In harmonic conditions, current contains different harmonics which each component can cause to create losses in fuse element. These losses lead to heat generation. If this heat is increased exceedingly, fuse element will be melted. Loss power can be written as:

$$
\begin{gathered}
P_{\text {Loss }}=R\left(I_{1}{ }^{2}+I_{2}{ }^{2}+\cdots+I_{h}{ }^{2}\right)=R I_{1}{ }^{2}\left(1+\sum_{h=2}^{n} \frac{I_{h}^{2}}{I_{1}^{2}}\right) \\
\Rightarrow R I_{1}{ }^{2}\left(1+T H D^{2}\right)
\end{gathered}
$$

Table 1. Electrical and thermal characteristic of different parts of fuse

\begin{tabular}{|l|c|c|c|c|c|c|c|}
\hline \multirow{2}{*}{ Parameter } & \multicolumn{7}{|c|}{ Material } \\
\cline { 2 - 8 } & $\begin{array}{c}\text { Ceramic } \\
\text { body (5) }\end{array}$ & $\begin{array}{c}\text { Copper } \\
(1,2)\end{array}$ & $\begin{array}{c}\text { Iron FE40 } \\
(3)\end{array}$ & $\begin{array}{c}\text { granular } \\
\text { quartz (7) }\end{array}$ & $\begin{array}{c}\text { Silver } \\
(6)\end{array}$ & $\begin{array}{c}\text { Insulation material } \\
\text { /pressed carton (4) }\end{array}$ & Air \\
\hline \multicolumn{1}{|c|}{ Density, $\rho\left[\mathrm{kg} / \mathrm{m}^{3}\right]$} & 2400 & 8900 & 7190 & 1500 & 8210 & 1400 & 1.09 \\
\hline $\begin{array}{l}\text { Specific heat, } \\
\mathrm{c}\left[\mathrm{J} / \mathrm{kg}^{\circ} \mathrm{C}\right]\end{array}$ & 1088 & 387 & 420.27 & 795 & 377 & 0.099 & 1006 \\
\hline $\begin{array}{l}\text { Thermal conductivity, } \\
\lambda\left[\mathrm{W} / \mathrm{m}^{\circ} \mathrm{C}\right]\end{array}$ & 1 & 385 & 52.028 & 0.325 & 121.22 & 0.063 & 0.027 \\
\hline
\end{tabular}

Research article

COIndian Society for Education and Environment (iSee)
"Power system fuses" http://www.indjst.org
H.F.Farahani \& M.Sabaghi Indian J.Sci.Technol. 
Fig. 5. Voltage distribution along the fuse element

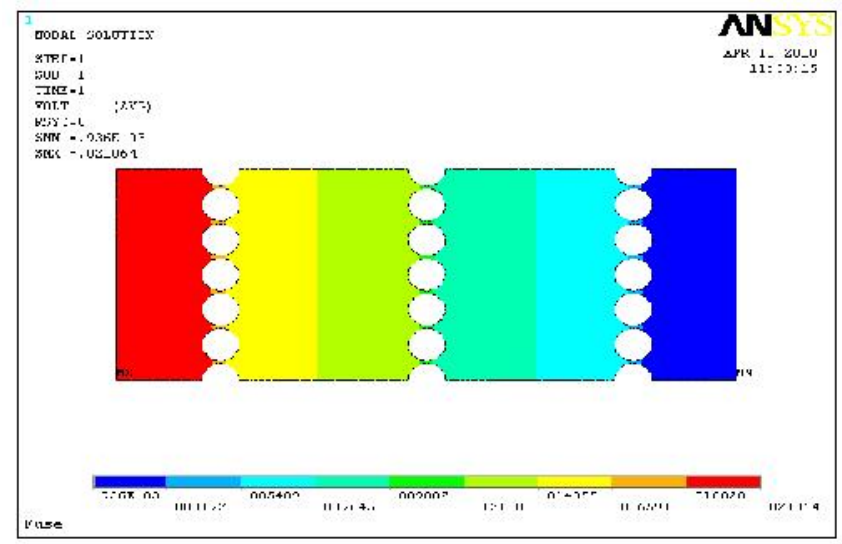

Fig. 6. Thermal flux along the fuse element

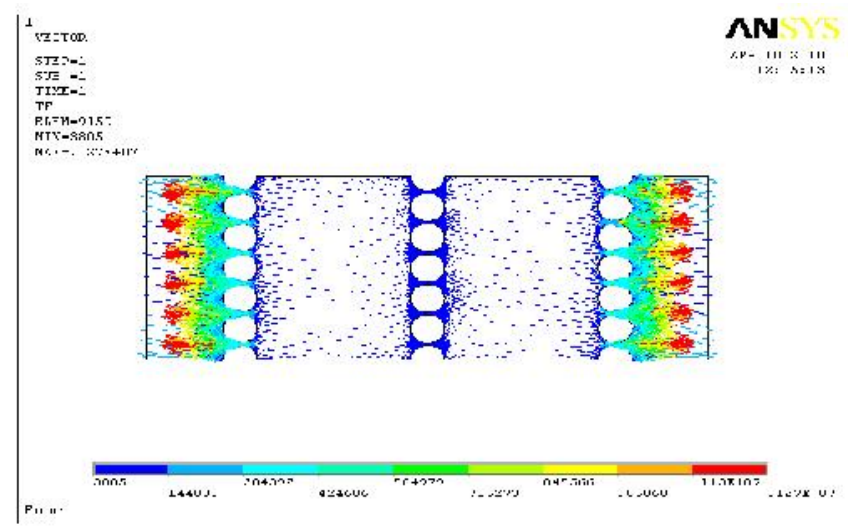

Fig. 7. Normalized power losses in fuse length versus frequency

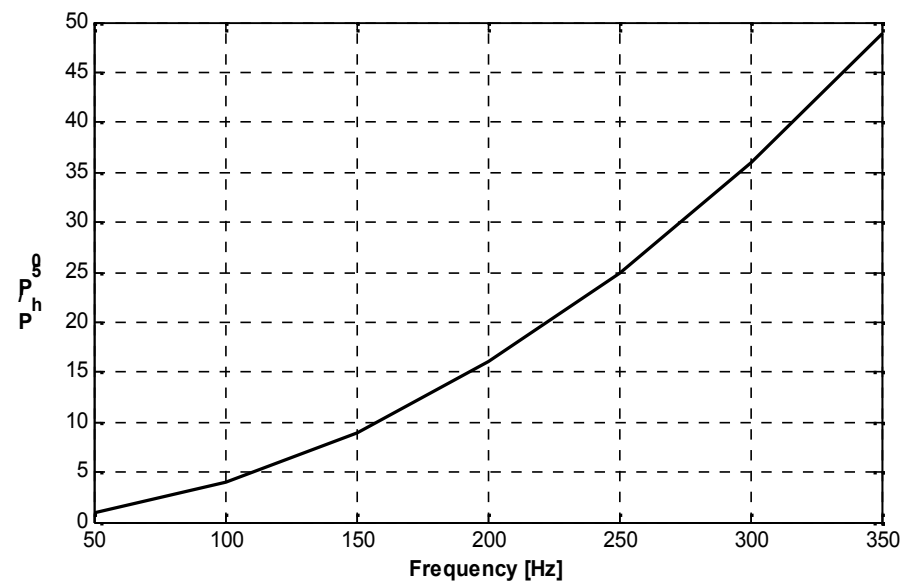

Fig. 8. Temperature distribution along the fuse element in harmonic condition

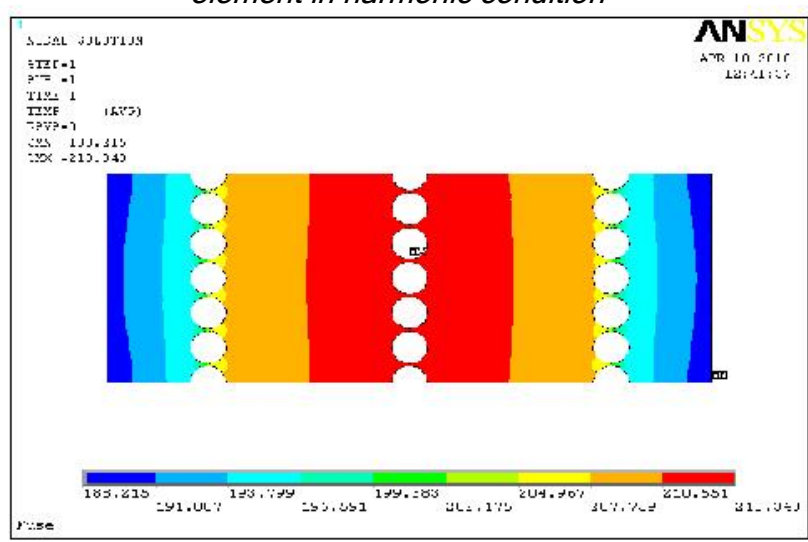

Fig. 9. Voltage distribution along the fuse element in harmonic condition

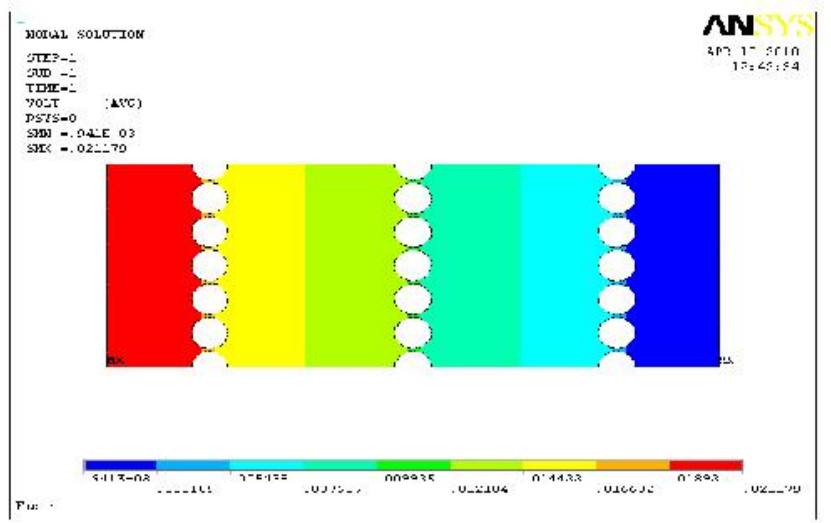

Fig. 10. Temperature distribution along the fuse link in harmonic condition ( $T H D=40 \%$ )

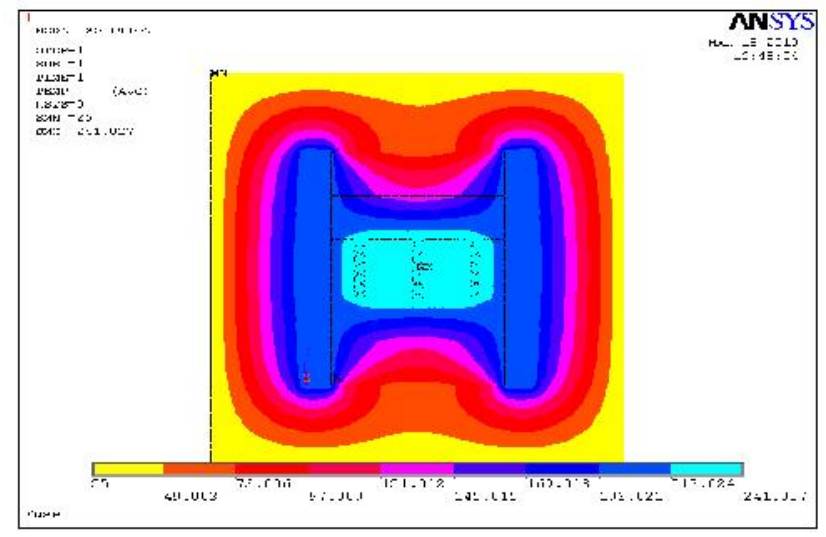


The nominal power losses (for sinusoidal current) of fuse can be written as:

$$
P_{r}=R I_{r}^{2}
$$

To have $P_{\text {loss }}=P_{r}$, using (1) and (2) can be conclude that:

$$
I^{*}=\frac{I_{r}}{\sqrt{1+T H D^{2}}}
$$

Where $I^{*}$ is the new nominal fuse current in harmonic condition. Fuse loss power is calculated for different harmonics with $\mathrm{I}=100 \mathrm{~A}$ which its results are listed in Table.2. From this table data, normalized loss power in fuse element versus frequency is shown in Fig. 7.

Table 2. Power losses in fuse in different harmonics

\begin{tabular}{|c|c|c|c|}
\hline $\mathrm{f}$ & $\mathrm{h}$ & $\mathrm{P}(\mathrm{W} / \mathrm{m})$ & $\mathrm{P}_{\mathrm{h}} / \mathrm{P}_{50}$ \\
\hline 50 & 1 & 0.721 & 1 \\
\hline 100 & 2 & 2.88 & 3.99 \\
\hline 150 & 3 & 6.49 & 9 \\
\hline 200 & 4 & 11.53 & 15.99 \\
\hline 250 & 5 & 18.02 & 24.99 \\
\hline 300 & 6 & 25.94 & 35.97 \\
\hline 350 & 7 & 35.3 & 48.95 \\
\hline
\end{tabular}

This figure indicates that this function is approximately quadratic which can be written as;

$$
\frac{P_{h}}{P_{1}}=0.0004 \times f^{2}+0.0002 \times f-0.013
$$

According to results from Table.2, an estimated equation can be expressed for normalized loss power in different harmonics versus harmonic orders:

$$
\frac{P_{h}}{P_{1}}=h^{2}
$$

Hence, harmonic of $h^{\text {th }}$ order creates $P_{h}=h^{2} P_{1}$ loss in fuse. If current amplitude of $h^{\text {th }}$ harmonic is considered $h^{2}$ times of fundamental component, following equation can be written:

$$
P_{\text {Loss }}=R\left(I_{1}^{2}+\sum_{h=2}^{\infty} h^{2} I_{h}^{2}\right)=R I_{1}^{2}\left(1+\sum_{h=2}^{\infty} h^{2} \frac{I_{h}^{2}}{I_{1}^{2}}\right)
$$

Regarding that harmonics cause to rise the fuse temperature, therefore, amplitude of current fundamental component should be decreased. In other words, it should be de-rated or its capacity should be decreased. If current fundamental component is equal to $I_{r}$, de-rated current value will be obtained from:

$$
I^{*}=\frac{I_{r}}{\sqrt{1+\sum_{h=2}^{\infty} h^{2} \frac{I_{h}^{2}}{I_{1}^{2}}}}
$$

For instance, fundamental component and its harmonics are considered as follow:

$$
I_{1}=100 \mathrm{~A}, I_{3}=10 \mathrm{~A}, I_{5}=5 \mathrm{~A}
$$

Then, power loss can be calculated in two cases:

- without considering skin effect

- considering skin effect

Not considering skin effect (ignoring skin effect)

By ignoring skin effect, power loss can be calculated from (1). RMS fuse current should be de-rated about $0.62 \%$. For Research article COIndian Society for Education and Environment (iSee) example, if fuse nominal current is $100 \mathrm{~A}$, current value of more than 99.38 A should not be flown through fuse:

$$
\begin{aligned}
& I^{*}=\frac{100}{\sqrt{1+\left(\frac{10}{100}\right)^{2}+\left(\frac{5}{100}\right)^{2}}}=99.38 \mathrm{~A} \\
& D R \%=\frac{99.38-100}{100} \times 100=-0.62 \%
\end{aligned}
$$

\section{Considering skin effect}

If frequency effect or skin effect is considered, power loss should be determined from (6). In this case, DR is about $6.85 \%$ which is 10 times greater than DR in previous case:

$$
\begin{gathered}
I^{*}=\frac{100}{\sqrt{1+9\left(\frac{10}{100}\right)^{2}+25\left(\frac{5}{100}\right)^{2}}}=93.15 \mathrm{~A} \\
D R \%=\frac{93.15-100}{100} \times 100 \approx-6.85 \%
\end{gathered}
$$

Fuse simulation results when $T H D=10 \%$

In this case, current THD through fuse is approximately equal to $10 \%$. In continue thermal behavior of fuse different parts are evaluated using obtained simulation results. Temperature distribution in fuse element is shown in Fig. 8.

Clearly in this figure, the hottest point of fuse is in center of this element. The least temperature is located in external edges which its value is $188.215{ }^{\circ} \mathrm{C}$. Voltage distribution along fuse element length is plotted in Fig. 9. According to this figure, the minimum and maximum potential of fuse element are respectively equal to $0.941 \mathrm{mV}$ and $21.179 \mathrm{mV}$. In this condition, voltage values of fuse contacts are considered $0 \mathrm{~V}$ and $22.12 \mathrm{mV}$.

\section{Fuse simulation results when $T H D=40 \%$}

Thermal distribution when $\mathrm{THD}=40 \%$ is shown in Fig. 10 that minimum and maximum temperatures are respectively $25.00{ }^{\circ} \mathrm{C}$ and $241.027{ }^{\circ} \mathrm{C}$ which in comparison with previous case, they are significantly increased.

THD effects on fuse temperature increasing

To investigate THD effects on fuse temperature, the hottest point temperature values for different THD values are obtained which are listed in Table 3.

Fuse temperature variation versus different THD values curve is specified in Fig. 11. This figure shows that by THD Table 3. Fuse hot point temperature in different THD

\begin{tabular}{|c|c|c|c|c|c|}
\hline THD & 0 & 10 & 20 & 30 & 40 \\
\hline $\mathrm{T}_{\text {hot }}$ & 211 & 213.3 & 218.65 & 228 & 240.8 \\
\hline
\end{tabular}

Fig. 11. Fuse hot point temperature versus $T H D$

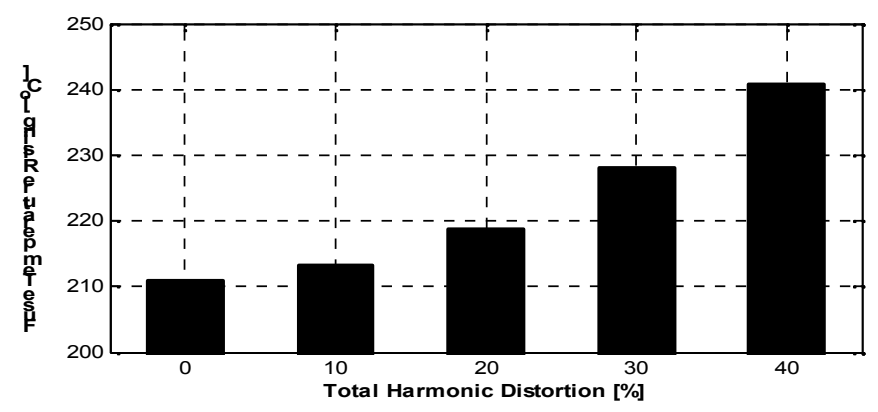


increasing, fuse temperature will be increased as well. This relationship can be estimated by following quadratic equation:

$$
T_{\text {hot }}=0.01 \times T H D^{2}+0.029 \times T H D+210
$$

\section{Conclusion}

In this paper, firstly, fuse operation has been analyzed. For this purpose, a sample fuse has been modeled in ANSYS software. Besides, regarding that fuse temperature has direct effect on fuse operation and its melting, therefore, fuse thermal analysis has been modeled and simulated in ANSYS. Moreover, fuse has been simulated under sinusoidal current and different parameters such as temperature distribution, thermal flux, voltage and so on relating to various parts of fuse have been obtained and investigated. Furthermore, an equation between fuse and harmonics has been presented. Also, fuse temperature increasing due to harmonic existing in fuse current has been evaluated. Fuse nominal current for different THD has been determined as well. Finally, a sample fuse under different harmonic currents has been simulated and the hottest point curve versus various THD values has been presented using simulation results by which fuse temperature at different THDs can be obtained.

Reference

1. Agarwal MS, Stokes AD (1987) Pre-arcing behaviour of open fuse wire. J. Physics D: Appl. Phys. 20(10), 12371242.

2. Baraboi A, Ciutea I, Adam M, Pancu C and Baraboi TA (1999) Modelling and simulation of the thermal stress at the electric fuses contacts. 6th Int. Conf. on Electrical Fuses \& their Appl. pp:59-64.

3. Beaujean DA, Newbery PG and Jayne MG (1995) Modelling fuse elements using a C.A.D. software package. Proc. of the 5th ICEFA, IImenau (Germany). pp:133-142.

4. Bottauscio O (1991) Influence of short-circuit power factor on the cuoff current of low voltage fuses. Conf. Electric Fuses \& Their Appl. (ICEFA) pp: 201-205.

5. Cañas C, Fernández L, et al. (1999) Minimum breaking current obtaining in fuses. Proc. of the 6th ICEFA, Torino (Italy).

6. Dolegowski M (1976) Calculation of the course of the current and voltage of a current-limiting fuse. Conf. Electric Fuses \& Their Appl (ICEFA). pp:218.

7. Douglas J (1993) Solving problems of power quality. EPR/ J. (Dec.)

8. Fernández L, Cañas C, Llobell J, Curiel J, Aspas J, Ruz J and Cavallé $F$ (1995) A model for pre-arcing behavior simulation of H.V. full-range fuse-links using the finite element method. Proc. of the 5th ICEFA, IImenau (Germany). pp:162-168.

9. Garrido C, and Cidrás J (1999) Study of fuselinks with different $\mathrm{t}-\mathrm{I}$ curves using a mathematical model. Proc. of the 6th ICEFA, Torino (Italy). pp: 21-24.

10. Gelet J, Tournier D and Ruggiero M (1999) Evaluation of thermal and electrical behaviour of fuses in case of paralleling and/or high frequencies. Proc. of the 6th ICEFA, Torino (Italy). pp: 49-53.

11. Gnanalingam S and Wilkins R (1980) Digital simulation of fuse breaking tests. Proc. IEE 127, 434-440.

12. Hoffmann $G$ and Kaltenborn $U$ (2003) Thermal modeling of high voltage H.R.C. fuses and simulation of tripping characteristic. 7th Int. Conf. on Electrical Fuses \& Their

Research article

Clndian Society for Education and Environment (iSee)

"Power system fuses" http://www.indjst.org
Vol. 5 No. 3 (Mar 2012) ISSN: 0974- 6846

Appl. pp:174-180.

13. Hoffmann G and Kaltenborn U (2003) Thermal modelling of high voltage H.R.C. fuses and simulation of tripping characteristic. Proc. of the 7th ICEFA, Gdansk (Poland). pp:174-180.

14. Jakubiuk K and W AW (2003) Heating of fuse-elements in transient and steady-state. Proc. of the 7th ICEFA,_Gdansk (Poland). pp:181-187.

15. John A (2006) Selection, application, and interchangeability of medium-voltage power fuses in motor control centers. IEEE Transact. on Industry Appl. 42(6) pp:?.

16. KawaseY and Miyatake T (2000) Heat analysis of a fuse for semiconductor devices protection using 3-D finite element method. IEEE Transact. on Magnetics. 36(4), 1377-1380.

17. Kürschner H, Ehrhardt $A$ and Nutsch G (1995) Calculation of prearcing times using the finite element method. Proc. Of the 5th ICEFA, IImenau (Germany). pp:156-161.

18. Lee S-H (2010) Application of high voltage current limiting fuse model using ATP-draw. Dielectrics \& Electrical Insulation, IEEE Transact. 17(6),1806-1813.

19. Lijun J, Zhiying M et al. (1999) The research on the stable temperaturerise of high-voltage current-limiting fuse-link. 6th Int. Conf. on Power Fuses and their Appl.

20. Lindmayer M (1999) 3D simulation of fusing characteristics including the M-Effect. Proc. of the 6th ICEFA,Torino (Italy). pp: $13-20$.

21. Memiaghe $S$, Bussière $W$ and Rochette $D$ (2007) Numerical method for prearcing times: Application in HBC fuses with heavy fault-currents. Conf. on Electric Fuses and Their Appl. (ICEFA). pp:127-132.

22. Narancic VN and Fecteau G (1984) Arc energy and critical tests for HV current limiting fuses. Conf. Electric Fuses \& Their Appl. (ICEFA). pp: 236-251.

23. Petit A, St-Jean G and Fecteau G (1989) Empirical model of a currentlimiting fuse using EMTP. IEEE Trans. Power Delivery 4, 335-341.

24. Plesca A (2007) A complete 3D thermal model for fast fuses. Blaise Pascal Academic Press ICEFA.

25. Pleşca A (2001) Overcurrent protection systems for power semiconductor installations. Ph.D. Thesis, laşi, Romania.

26. Pleşca A (2003) Thermal simulations of fast fuses for power semiconductor devices protection. Proc. of the 7th ICEFA, Gdansk (Poland). pp: 200-205.

27. Rochette D, Bussière W. et al. (2007) Modelling of the prearcing period in HBC fuses including solid-liquid- vapour phase changes of the fuse element. Conf. on Electric Fuses \& Their Appl. (ICEFA). pp: 87-93.

28. Sasu M (1995) Simulation of the heat-transfer phenomena in electrical fuses by means of the digital computer. Eur. Transact. on Electrical Power Eng. 5(3), 187-191.

29. Torres E, Fernandez E, Mazon AJ, Zamora I, Perez JC (2005) Thermal analysis of medium voltage fuses using the finite element method. Power Tech. 2005 IEEE, Russia: $\mathrm{pp}: 1-5$.

30. Wilkins R (1991) Steady-state current sharing in fuses with asymmetrical arrangements. Proc. of the 4th ICEFA Nottingham (UK). pp: 28-33.

31. Wilniewczyc M, McEwan PM and Crellin D (1999) "Finiteelement analysis of thermally-induced film de-bonding in single and two-layer thick-film substrate fuses." Proc. of the 6th ICEFA, Torino (Italy): 29-33. 